Article

\title{
Towards Smart Energy Grids: A Box-Constrained Nonlinear Underdetermined Model for Power System Observability Using Recursive Quadratic Programming
}

\author{
Nikolaos P. Theodorakatos ${ }^{1}$, Miltiadis Lytras ${ }^{2,3, *(D)}$ and Rohit Babu ${ }^{4}$ \\ 1 School of Electrical and Computer Engineering, National Technical University of Athens (NTUA), \\ Iroon Polytechneiou 9, 15780 Zografou, Athens, Greece; nikos.theo2772002@gmail.com \\ 2 School of Business and Economics, Deree-The American College of Greece, Gravias 6, \\ 15342 Aghia Paraskevi, Greece \\ 3 Effat College of Engineering, Effat University, P.O. Box 34689, Jeddah 21478, Saudi Arabia \\ 4 Department of Electrical \& Electronics Engineering, Bharat Institute of Engineering and Technology, \\ Mangalpally, Ibrahimpatnam, Ranga Reddy, Hyderabad, Telangana 501510, India; rohitbabu@biet.ac.in \\ * Correspondence: mlytras@acg.edu
}

Received: 20 February 2020; Accepted: 31 March 2020; Published: 4 April 2020

check for updates

\begin{abstract}
This paper introduces an underdetermined nonlinear programming model where the equality constraints are fewer than the design variables defined on a compact set for the solution of the optimal Phasor Measurement Unit (PMU) placement. The minimization model is efficiently solved by a recursive quadratic programming (RQP) method. The focus of this work is on applying an RQP to attempt to find guaranteed global minima. The proposed minimization model is conducted on IEEE systems. For all simulation runs, the RQP converges superlinearly towards optimality in a finite number of iterations without to be rejected the full step-length. The simulation results indicate that the RQP finds out the minimal number and the optimal locations of PMUs to make the power system wholly observable.
\end{abstract}

Keywords: smart power transmission system; synchronized measurements; optimal PMU placement; underdetermined nonlinear systems; algorithms; smart energy grids; smart cities

\section{Introduction}

Phasor measurement unit (PMU) is a metering device that can provide real-time voltage and current synchrophasor measurements with high accuracy. PMUs play an imperative role in state estimation, monitoring, protection and wide area control in power systems [1]. Due to cost reasons, a full deployment of PMUs in the network is not realistic. One of the most important addressed issues is the strategic choice of the minimum number and locations of PMUs, ensuring complete network observability. Therefore, the optimal PMU placement (OPP) problem should be solved in order to make the power system completely observable by optimally placed PMUs at network buses [2,3].

Deterministic and stochastic algorithms are implemented for the solution of the OPP problem [2,3]. Several deterministic algorithms have been published in the literature for the solution of the OPP problem. The dominant optimization method regarding the OPP problem is the Binary Integer Linear Programming (BILP) technique. The BILP model requires the minimization of the objective function with inequality constraints and binary-valued decision variables [4-10].

A Weighed Least Square (WLS) algorithm is developed in [11] for the OPP problem. Authors of [12] proposed a nonlinear programming model for the solution of the OPP problem. In [13-15] the 
OPP problem is unraveled using the mixed-integer semi-definite programming approach subjected to linear matrix inequality. This technique is based on numerical observability, whereas most of the other techniques are based on topological observability, which may or may not ensure numerical observability to be executed successfully for state estimation. In [16,17], mixed-integer linear programming and nonlinear programming techniques are compared to check their suitability for networks of different sizes.

Stochastic algorithms have also been used for the OPP problem. A recursive tabu search is suggested in [18], which has been claimed superior to multiple Tabu search and higher observability. Binary particle swarm optimization (BPSO) algorithm is developed for the solution of the OPP problem in [19]. In [20], the authors proposed a BPSO algorithm to minimize the number of substations in which installations must be performed for making all voltage levels observable while being subject to various practical constraints. Authors of [21] proposed the binary gravitational search algorithm to solve the OPP problem. Authors in [22], presented a modified binary Cuckoo optimization algorithm for the solution of the OPP problem with maximum redundancy. Genetic algorithms (GA) have been applied to determine the optimal allocation of PMUs in [23,24].

Accurate knowledge of transmission system parameters, such as series impedance, optimizes distance relay settings and impedance-based fault location. A new method is developed to measure transmission line impedances and admittances from synchronized phasor measurements in [25].

A new on-line method for estimating transmission line constants of a power system is proposed in [26]. In [27], a new State Estimation (SE) framework is proposed for processing Remote Terminal Unit (RTU) and PMU measurements separately in order to leave the traditional weighted least square (WLS)-based SE software unchanged. Phasor measurement unit based fault location techniques are proposed in [28-30]. Other methods, such as an optimized extreme learning machine-based approach, use synchrophasors to ensure real-time power transient stability prediction [31].

Up to now, a BILP model is written to its standard form in which the number of the constraints is equal to the number of optimization variables for the OPP problem [4-10]. This work introduces an underdetermined system of nonlinear equations, where the equations are fewer than the design variables for the solution of the OPP problem. When a significant fraction of the optimization problem constraints are eliminated, the feasibility diagnosis is accelerated and speeds up the solution algorithm in finding the optimality [32-36]. Thus, a reduction in problem size typically translates to a reduction in total running time in comparison with past studies such as $[11,12,17]$.

The solution to such underdetermined systems is based on the Recursive Quadratic Programming (RQP) method [35]. The contribution of this work is fourfold as follows:

- The proposed nonlinear model is solved using a Recursive Quadratic Programming (RQP) method with super-linear convergence properties avoiding the Maratos effect.

- The innovation of the local search procedure is that the RQP converges super-linearly towards optimality, satisfying the binary restriction.

- $\quad$ The RQP presents a fast convergence rate towards optimality.

- The RQP method delivers multiple optimal solutions in a reasonable time with those consumed by a BILP model being solved by the branch-and-bound method (BBM).

The remainder of this paper is organized as follows. Section 2 presents the basics of the Recursive Quadratic Programming (RQP) method. Section 3 formulates the OPP problem as an RQP optimization model. Section 4 gives an overview of computational results, whereas a performance evaluation of the proposed RQP algorithm for the solution of the OPP problem is carried out in Section 5. Finally, Section 6 concludes the paper. 


\section{A Recursive Quadratic Programming Background}

The Recursive Quadratic Programming Algorithm solves nonlinear optimization problem of the form [35]:

$$
\begin{aligned}
& \min _{x \in \mathfrak{R}^{n}} J(x) \\
& \text { s.t }\left\{\begin{array}{l}
G_{i}(x)=0, i=1, \ldots, M_{e} \\
G_{j}(x) \leq 0, j=M_{e}+1, \ldots, M
\end{array}\right.
\end{aligned}
$$

The RQP methods belong to the most frequently used algorithms for the solution of practical optimization problems due to their robustness and their good convergence properties. RQP methods can be proven to achieve global convergence and locally superlinear convergence rate; even locally quadratic convergence can be attained if the Hessian of the Lagrangian $\nabla_{x x}^{2} L(x, \lambda)$ is available. Note that, the Lagrangian is given by:

$$
L(x, \lambda)=J(x)+\lambda^{T} G(x)
$$

where $\lambda \in \mathfrak{R}^{m}$ is the vector of Lagrange multipliers.

The fundamental principle of RQP methods is to find Karush-Kuhn-Tucker (KKT) points, i.e., points that satisfy the necessary optimality conditions [35]. RQP methods mainly apply Newton's method to find zeros of

$$
\nabla_{x} L(x, \lambda)=\nabla_{x} J(x)+(\lambda)^{T} \nabla_{x} G(x)
$$

that satisfy the constraints $G$, using a local linearization.

In this basic form, quadratic subproblems of the form

$$
\begin{aligned}
& \min _{d \in \mathfrak{R}^{n}} \frac{1}{2} \nabla_{x x}^{2} L(x, \lambda) d+\nabla_{x} J(x) d \\
& \text { subject to }\left\{\begin{array}{l}
G_{i}(x)+\nabla_{x} G_{i}(x) d=0, i=1 \ldots M_{e} \\
G_{j}(x)+\nabla_{x} G_{j}(x) d \leq 0, i=M_{e}+1 \ldots M
\end{array}\right.
\end{aligned}
$$

are solved to obtain a search direction $d$.

Often, the Hessian of the Lagrangian $\nabla_{x x}^{2} L(x, \lambda)$ is replaced by an update of Broydon-Fletcher -Goldfarbo-Shanno (BFGS) type, which has the additional benefit that only strictly convex quadratic programs have to be solved [35]. The computation of derivatives is a crucial element in nonlinear optimization. Mainly the first derivatives, i.e., the gradient of the objective function and the Jacobian of the constraints, is necessary to find a descent direction. The RQP is an iterative process that defines a series of points $x^{[k]}$ (called iterates) that (ideally) converge to an optimal point. It consists very roughly of the following steps [35]:

1. Check termination criteria: For testing an iterate $x^{[k]}$, the first-order optimality conditions KKT have to be evaluated.

2. Solve approximate the QP sub-problem.

3. Use the solution from 2, to define a new iterate employing a merit function to find a suitable step-length.

Merit functions are needed to obtain a scalar measure of goodness of the trial point

$$
x^{[k+1]}=x^{[k]}+a^{[k]} d^{[k]}
$$

which is an update of the current iterate $x^{[k]}$ by the result $d^{[k]}$ of Esq. QP and a scalar step-length $a^{[k]}$ that is introduced to achieve global convergence (since RQP is derived from Newton's method that also depends on line search for globalization). The merit function is given by [35]:

$$
L(x ; n) \triangleq J(\vec{x})+\sum_{i=1}^{M e} n_{i} \times\left|G_{i}(\vec{x})\right|+\sum_{i=M e+1}^{M} n_{i} \max \left\{0, G_{i}(x)\right\}
$$


After determining the search direction $d^{[k]}$ from the QP, we have to find a suitable step-length $a^{[k]}$. The line search procedure can be used to determine a step-length parameter $a^{[k]}$ based on the Armijo rule. The step-length is chosen to yield a sufficient decrease of a suitable merit function, which measures progress towards optimality [35]. Figure 1 shows RQP's flowchart.

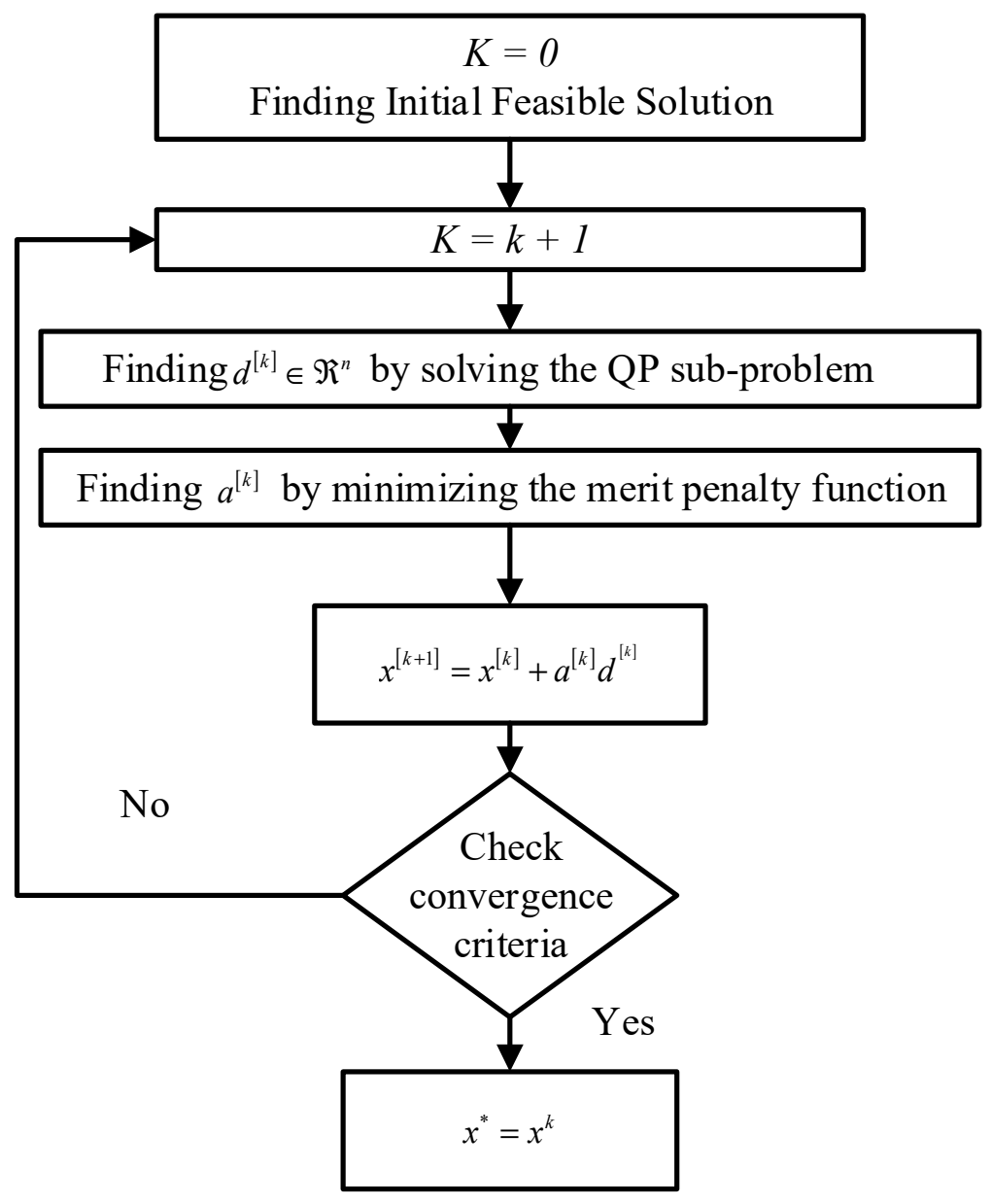

Figure 1. Flowchart of recursive quadratic programming.

A difficulty in the context of RQP methods is that the line search procedure may prevent the full step from being taken [37]. The step-length $a \in(0,1]$ is chosen to yield a sufficient decrease of a suitable penalty function, which measures progress towards optimality [37]. The convergence rate depends upon the reduction on the penalty function at each iteration, which relies on the choices of the descent directions and the step-length $a$ [37]. The penalty function may not allow a unity step-length near the solution and thus may prevent q-superlinear convergence [37].

This phenomenon is called as the Maratos effect [37]. By using the RQP method through the fmincon of MATLAB optimization toolbox [38], the unity step-length is being taken decreasing the nonsmooth exact penalty function whenever the current iterate is sufficiently close to a local minimum point. Thus, the Maratos effect can be obviated, ensuring superlinear convergence to the optimum point. The RQP is converged when the difference between the current objective value and the previous objective value is less than the optimality tolerance [35].

Additionally, the constraints are satisfied within the feasibility tolerance, whereas step-tolerance specifies the termination tolerance for the design variable [36]. Therefore, the termination is succeeded based on the tolerance criteria in terms of objective evaluation, maximum constraint violation and first-order optimality [36]. 


\section{Optimum Design Model Formulation}

Let the continuous decision variables $x_{i}$ denote the presence $\left(x_{i}=1\right)$ or absence $\left(x_{i}=0\right)$ of PMU at bus $i$. The OPP problem is formulated as a nonlinear minimization model [12]:

$$
\begin{gathered}
\min _{\vec{x} \in R^{n}} J(\vec{x})=\vec{x}^{T} \cdot W \cdot \vec{x}=\sum_{i=1}^{n} w_{i} \cdot x_{i}^{2}, W \in R^{n \times n}=I_{n} \\
\text { s.t. }\left\{\begin{array}{l}
f(x)=\hat{0} \\
\hat{0} \leq x \leq \hat{1}
\end{array}\right.
\end{gathered}
$$

where, $x=\left(x_{1}, \cdots, x_{n}\right)^{T}$ is a decision variable vector, whose entry $x_{i}$ is set to either 1 or 0 to denote the presence or absence of a PMU at bus $i, W=\operatorname{diag}\left(w_{1}, \cdots, w_{n}\right)$ is the PMU cost or weight matrix, $n$ is the number of buses, $\hat{0}, \hat{1}$ is a vector whose entries are all zeros and ones, respectively, and $f(x)$ is a vector function whose ith entry defines an observability constraint for the ith bus [11,12]:

$$
f_{i}(x)=\left(1-x_{i}\right) \prod_{j \in \mathcal{N}_{i}}\left(1-x_{j}\right)=0, \forall i \in \mathcal{N}
$$

where $\mathcal{N}$ is the set of all buses and $\mathcal{N}_{i}$ is the set of buses adjacent to bus $i$. Each Equality Constraint (3) implies that at least one PMU should be installed at any one of the buses $i$ and $j \in \mathcal{N}_{i}$ in order to make bus $i$ observable. The optimal values of decision variables $x_{i}$ will be 1 or 0 as it has been proved in [12]. The above minimization model is reformulated and extended to employ with the underdetermined case.

Since we want to solve problems with a large number of constraints, many of them maybe are redundant [33,34]. The constraint redundancy gives rise to Jacobian rank deficiency, i.e., a singularity in iterative estimates of the constraint-set [34]. The preprocessing phase aims to simplify a given optimization problem by detecting and removing redundant constraints [33,34]. The preprocessing phase is applied to the primary constraint function to remove redundancies from the given constraints $[33,34]$. A redundant constraint is not required to define the boundaries of the feasible set, so the solution space is not affected. When the redundant constraints are dropped, the feasible region is expanded and more feasible solutions are located, whereas more constraints shrink the feasible region [35]. Since the enlarged feasible region involves more feasible points than the feasible region of the original model, more local minima are detected.

If a pre-solve procedure is applied after the model has been built, but before solving it, then a reduction in size can be achieved [33]. During the pre-solve process, an entirely new optimization problem is constructed. After the algorithm model has been optimized, the optimal solution is valid for the original problem [34]. 
Illustrative Example of Deletion Presolve Using the IEEE-14 Bus System

The size-reducing transformation, which leads to an underdetermined nonlinear model, is shown using the IEEE-14 bus system (Figure 2). The observability constraint function $f: R^{n} \rightarrow R^{n}$ in case of the IEEE 14-bus system is as follows [12]:

$$
f(\vec{x})=\left\{\begin{array}{l}
f_{1}=\left(1-x_{1}\right)\left(1-x_{2}\right)\left(1-x_{5}\right)=0 \\
f_{2}=\left(1-x_{2}\right)\left(1-x_{1}\right)\left(1-x_{3}\right)\left(1-x_{4}\right)\left(1-x_{5}\right)=0 \\
f_{3}=\left(1-x_{3}\right)\left(1-x_{2}\right)\left(1-x_{4}\right)=0 \\
f_{4}=\left(1-x_{4}\right)\left(1-x_{2}\right)\left(1-x_{3}\right)\left(1-x_{5}\right)\left(1-x_{7}\right)\left(1-x_{9}\right)=0 \\
f_{5}=\left(1-x_{5}\right)\left(1-x_{1}\right)\left(1-x_{2}\right)\left(1-x_{4}\right)\left(1-x_{6}\right)=0 \\
f_{6}=\left(1-x_{6}\right)\left(1-x_{5}\right)\left(1-x_{11}\right)\left(1-x_{12}\right)\left(1-x_{13}\right)=0 \\
f_{7}=\left(1-x_{7}\right)\left(1-x_{4}\right)\left(1-x_{8}\right)\left(1-x_{9}\right)=0 \\
f_{8}=\left(1-x_{8}\right)\left(1-x_{7}\right)=0 \\
f_{9}=\left(1-x_{9}\right)\left(1-x_{4}\right)\left(1-x_{7}\right)\left(1-x_{10}\right)\left(1-x_{14}\right)=0 \\
f_{10}=\left(1-x_{10}\right)\left(1-x_{9}\right)\left(1-x_{11}\right)=0 \\
f_{11}=\left(1-x_{11}\right)\left(1-x_{6}\right)\left(1-x_{10}\right)=0 \\
f_{12}=\left(1-x_{12}\right)\left(1-x_{6}\right)\left(1-x_{13}\right)=0 \\
f_{13}=\left(1-x_{13}\right)\left(1-x_{6}\right)\left(1-x_{12}\right)\left(1-x_{14}\right)=0 \\
f_{14}=\left(1-x_{14}\right)\left(1-x_{9}\right)\left(1-x_{13}\right)=0
\end{array}\right.
$$

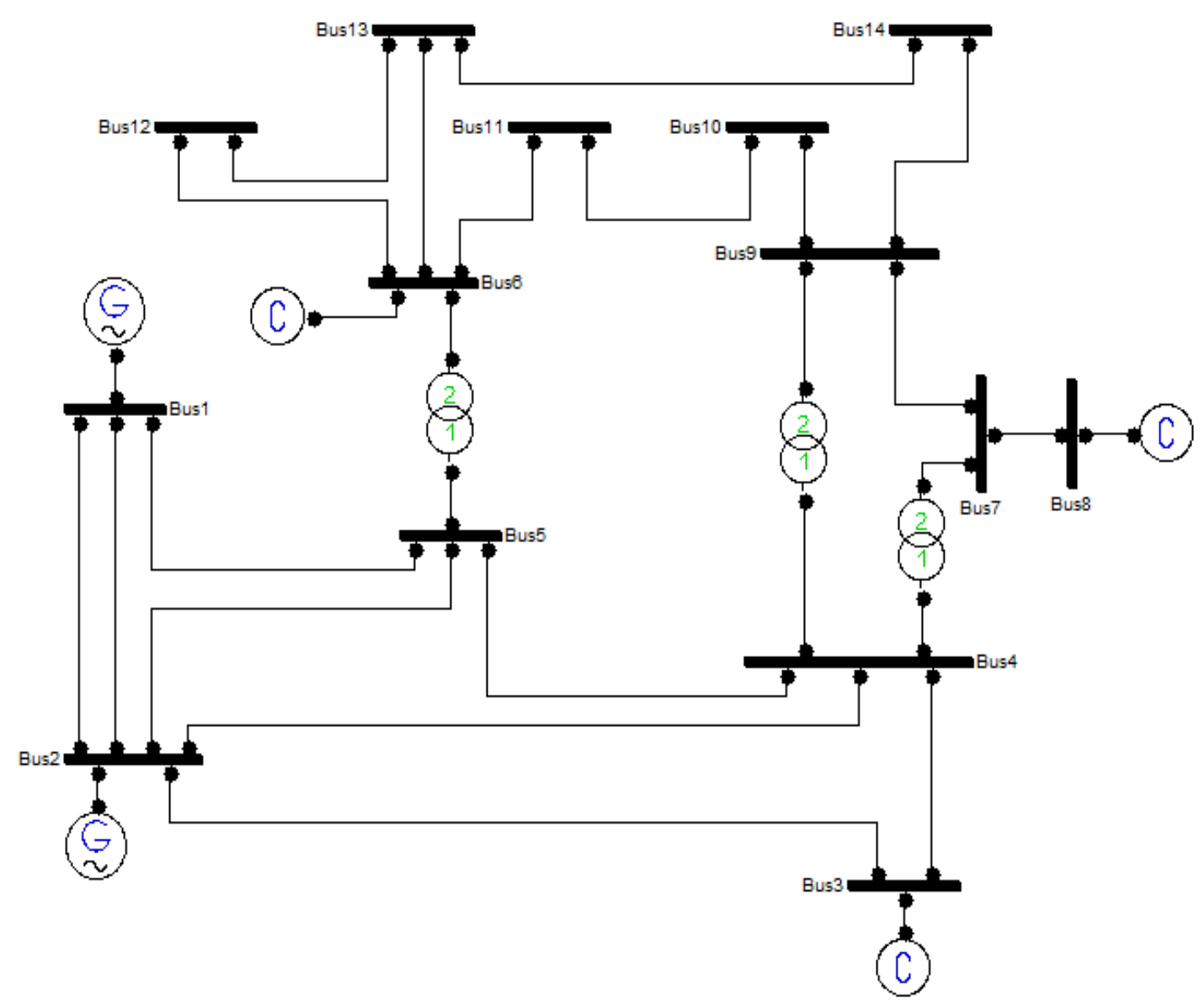

Figure 2. IEEE-14-bus.

As can be observed, some equality constraints are dependent on others and they are redundant constraints for the system of concern. A redundant constraint can be chosen as the one to be eliminated, 
for it can be reconstructed from those remaining [33]. Such a non-binding constraint could just as well be left out of the model, as this would not affect the optimum point [33].

For example, $f_{2}$ is obtained by multiplying $f_{1}=0$ by additional terms yielding the Esq. $\left(1-x_{3}\right)\left(1-x_{4}\right) f_{1}=0$. The $f_{2}$ is unnecessary since the constraint is always satisfied i.e., $\left\{f_{i}=0\right\}$ and it is removed. When a constraint is satisfied $\left\{f_{i}=0\right\}$, it can be removed from the system of interest. Let us consider the equality constraints $\left\{f_{12}, f_{13}\right\}$ so that:

$$
\left\{\begin{array}{l}
f_{12}=\left(1-x_{12}\right)\left(1-x_{6}\right)\left(1-x_{13}\right)=0 \\
f_{13}=\left(1-x_{13}\right)\left(1-x_{6}\right)\left(1-x_{12}\right)\left(1-x_{14}\right)=0
\end{array} \rightarrow f_{12}\left(1-x_{14}\right)=0 \rightarrow f_{13}\right. \text { is always satisfied }
$$

The product $f_{13}$ is resulting by multiplying the equation $f_{12}$ with an additional term and thus, it is always satisfied. Then, the constraint function involves only mutually independent constraints expanding the feasible region without changing the solution structure. The constraint function is as:

$$
h(\overrightarrow{\vec{x}})=\left[h_{1}(\vec{x}), h_{2}(\vec{x}), \ldots, h_{m}(\vec{x})\right]^{T}=0
$$

where the constraint function $h: R^{n} \rightarrow R^{m}(\mathrm{n}>\mathrm{m})$ is considered to be an underdetermined system of equations defined on a Box Constraint set, that is, $\vec{x} \in[0,1][35]$.

$$
h(\vec{x})=\left\{\begin{array}{l}
h_{1}=\left(1-x_{1}\right)\left(1-x_{2}\right)\left(1-x_{5}\right)=0 \\
h_{2}=\left(1-x_{2}\right)\left(1-x_{3}\right)\left(1-x_{4}\right)=0 \\
h_{3}=\left(1-x_{7}\right)\left(1-x_{8}\right)=0 \\
h_{4}=\left(1-x_{4}\right)\left(1-x_{7}\right)\left(1-x_{9}\right)\left(1-x_{10}\right)\left(1-x_{14}\right)=0 \\
h_{5}=\left(1-x_{9}\right)\left(1-x_{10}\right)\left(1-x_{11}\right)=0 \\
h_{6}=\left(1-x_{6}\right)\left(1-x_{10}\right)\left(1-x_{11}\right)=0 \\
h_{7}=\left(1-x_{6}\right)\left(1-x_{12}\right)\left(1-x_{13}\right)=0 \\
h_{8}=\left(1-x_{9}\right)\left(1-x_{13}\right)\left(1-x_{14}\right)=0
\end{array}\right.
$$

A solution that verifies the nonlinear system of equations and optimizes the quadratic objective function has been determined.

$$
J(\vec{x})=\sum_{i=1}^{n} w_{i} \cdot x_{i}^{2}\left(w_{i}=1\right)
$$

The optimal solutions derived by the RQP are $\{2,6,8,9\},\{2,6,7,9\},\{2,7,11,13\},\{2,7,10,13\}$, $\{2,8,10,13\}$ satisfying the equality constraints. Each optimum point is a feasible solution satisfying the equality constraints. The constraint function for different size power systems is as follows: $\Omega=\left\{0 \leq x_{i} \leq 1 ; i=1 \ldots n \mid h_{l}(\vec{x})=0 ; l=1 \ldots m\right\},\{n=14,30,57,118,300\},\{m=8,21,52,91,238\}$.

\section{Simulation Results and Discussion}

In this section, we solve the proposed nonlinear model by using the RQP method. To prove the effectiveness of the RQP to achieve optimality, the obtained objective value is compared to the one found by solving the Integer Linear Programming (ILP) model with binary-valued variables for each benchmark test system. The pure ILP model is solved by using the BBM. In this method, a relaxed continuous linear programming (LP) problem is formulated by ignoring the integer constraints. The relaxed problem is comprised of continuous variables. If the solution to the above relaxed continuous problem contains only involves integers, it is the optimal solution. If the solution has non-integer variables, one non-integer variable is selected and two sub-problems (two branches) are generated [39,40]. For one of them, a ceil constraint is added to the selected non-integer variable and, for the other branch, a floor constraint is added to it. If a branch has no feasible solutions, this branch is terminated $[39,40]$. If the solution only has integers, it becomes a candidate for the final optimal solution. If the solution has non-integer values, the process of the branching is again solved with these 
additional constraints. Once the branching process is completed for all the variables, all the integer solutions obtained by the difference branches are compared. The best solution is considered the final optimal solution as shown in the flowchart of Figure 3.

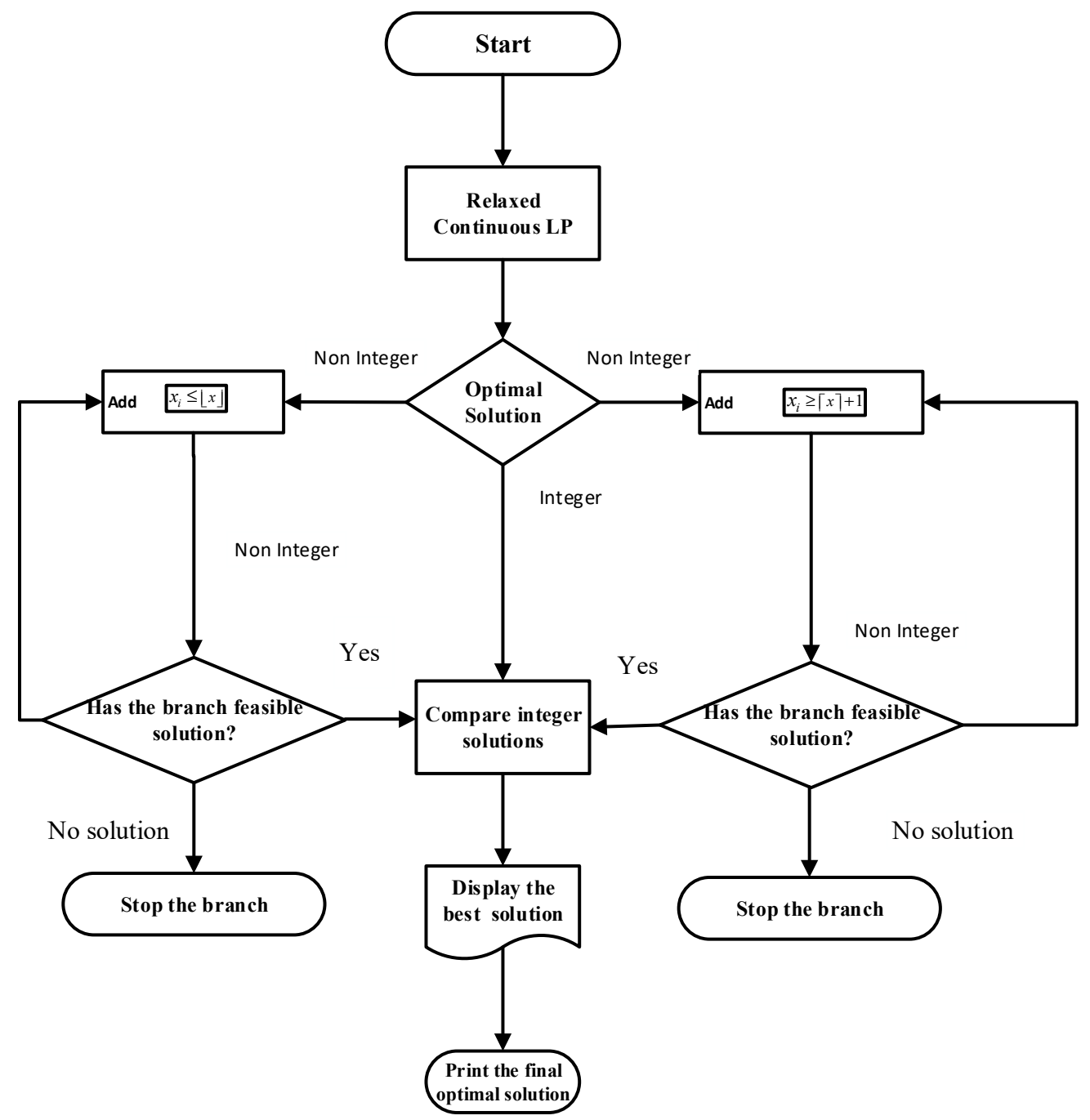

Figure 3. Flowchart of branch-and-bound method.

Several MILP routines implement the static-BBM, namely a BBM, including Cut Generation and Heuristics approaches via MATLAB intlinprog optimizer [38], a spatial BBM implemented by solving constraint integer programs (SCIP) [41], an LP-BBM using a primal-and-dual simplex implemented by MOSEK [42] and Gurobi optimizer [43]. Starting from the initial default point, MILP solvers can get only one optimal solution. This is because MILP employs the BB algorithm, and the solution is only updated when a better one is found. This means that solutions with the same results (same objective function value) are overlooked. Without considering other possible optimal solutions, the existing MILP solver stops searching and delivers the result when one optimal solution is found $[39,40]$.

The optimal solutions derived by static-BBM are listed in Tables A1 and A2 of Appendix A for each IEEE bus system [44]. As observed, each static-BBM results in a different optimal solution with the same number of PMUs for each IEEE bus system [44]. On the other hand, the RQP solves the proposed nonlinear program. The RQP is a gradient-based algorithm implemented through a fmincon optimizer routine [38]. To accelerate the convergence towards optimality, the gradient's vectors are given analytically to the optimizer routine [38]. The RQP algorithm model is applied to standard IEEE 
test systems [44]. The simulation results indicate that the RQP detects the minimum number and the optimal locations of PMUs to make the power system completely observable.

Now let us apply the RQP algorithm to the IEEE-300 system [44]. The nonlinear program is as follows:

$$
\min _{\vec{x} \in R^{n}} J(\vec{x})=\vec{x}^{T} . \text { W. } \vec{x} ; \text { s.t. } \vec{x} \in[0,1] \mid g: R^{n} \rightarrow R^{m}, m<n, m=238, n=300
$$

We set an arbitrary initial point through MATLAB command "rand" [38]. Then, the RQP generates the exact solution after 36 iterations, as shown in Table A3 of the Appendix A. The most notable is that no constraint violation exists. The numerical results displayed in Table A3 include the number of iterations, the total number of function evaluations, the convergent sequence of points $\left\{x_{k}\right\}_{k=0}^{\infty} \rightarrow x^{*}$ to the optimum point and the optimal objective function value. The termination is succeeded based on the design criteria in terms of objective function evaluation, the measured first-order optimality at the solution point and the calculated constraint violation. Table A3 illustrates that the step-length is fully being taken to enforce global convergence to the optimum point. As a consequence of that, the unit step-length is accepted so that the Maratos effect does not occur, ensuring a superlinear convergence rate to that optimum point.

Also, the norm of step and the first-order optimality are presented. During the RQP's iterations, the penalty function maintains the feasibility at the solution point. The objective function is minimized on the product $x^{*}$ in running time equal to $17.796910 \mathrm{sec}$ whereas the solution point satisfies the constraint function. The nonlinear model is computationally efficient in finding the required PMUs for the IEEE-300 bus system, as shown in Table A3 of Appendix A and Figure 4.
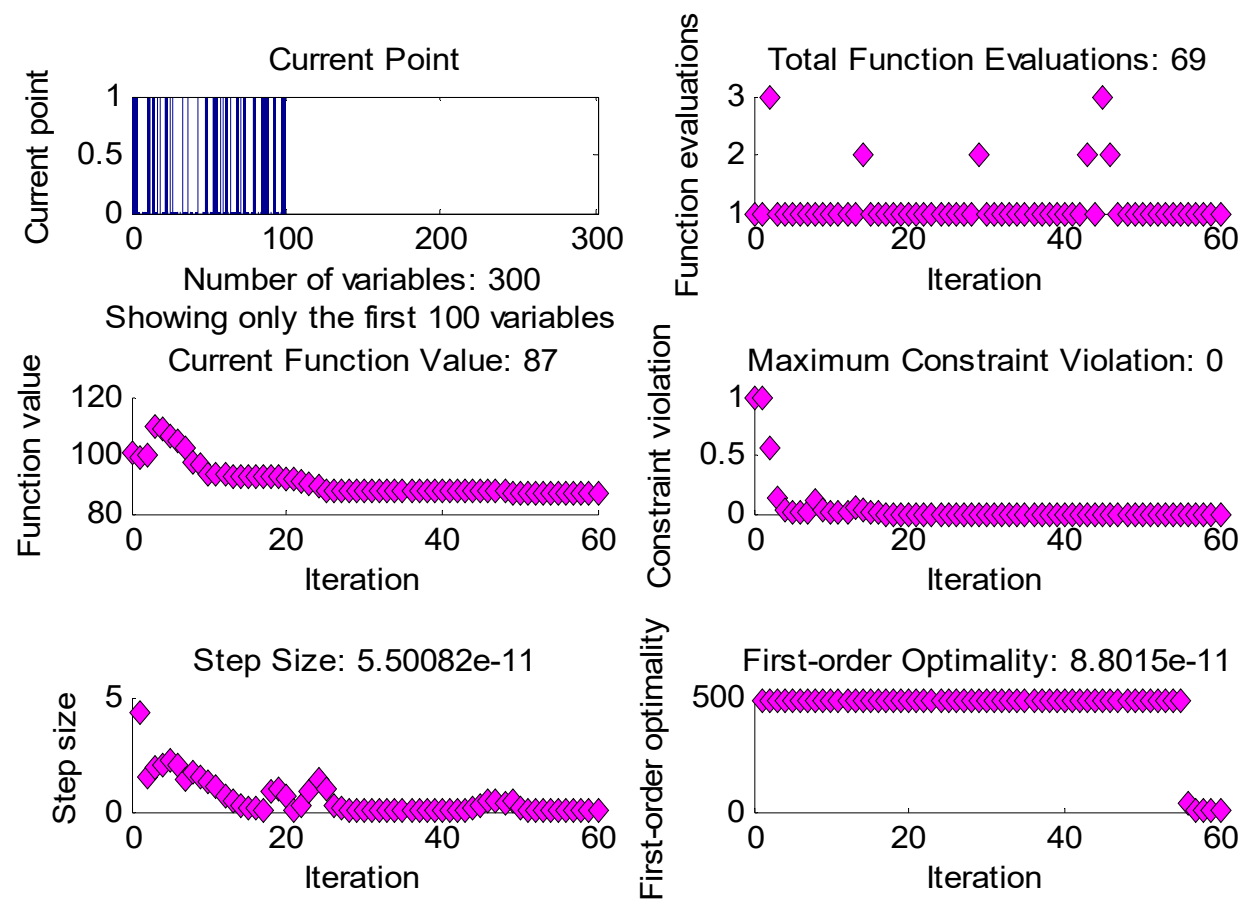

Figure 4. Convergence characteristics of RQP for the solution of the underdetermined nonlinear model.

The function evaluations lead to a feasible global solution point, whereas the constraints are satisfied within tolerances. The fmincon/RQP optimizes the tuning parameters i.e., TolX, TolFun, and TolCon to converge to global optimality whereas the binary restriction $\vec{x} \in\{0,1\}^{n}$ is satisfied.

The optimization is completed because the relative first-order optimality measure, $8.8015 \times 10^{-11}$, is less than the selected tolerance TolFun. Additionally, step-tolerance TolX specifies the termination tolerance for the design variable, as shown in Figure 4. Note that TolFun is a tolerance for both: the 
size of the latest change in the objective function value and the value of the first-order optimality measure [38]. The RQP detects a feasible solution satisfying all of the constraints in which the objective function takes it's a minimal value, whereas the Euclidean norm of step is less than $5.50082 \times 10^{-11}$ (Figure 4). The norm of step is the current step-size, that is the last displayment in $x$. Therefore, the termination is succeeded based on the tolerance criteria in terms of objective evaluation, maximum constraint violation i.e., TolCon results in zero and first-order optimality [38].

The RQP identifies alternative solution points over the feasible region constituted by the design variable bounds and constraints, as shown in Table A4 of Appendix A. The number of function evaluations measures the convergence rate [35]. Provided that the function evaluation is the most time-consuming part of the algorithm, this process characterizes the convergence speed [35]. Using approximations differences, the RQP detects the solution point but takes longer to achieve optimality as shown in Table A5 of the Appendix A. The best performance would be obtained by combining analytically given gradient constraints with the RQP method [35].

The RQP requires only a small number of iterations and function evaluations to terminate successfully. The elapsed time overall outcomes by RQP are comparable to those spent by BBM, as shown in Table A5 of the Appendix A.

\section{Performance Evaluation and Comparisons}

In this paper, for unraveling the OPP problem, a minimization model is constructed in which the objective function is minimized under an underdetermined nonlinear system of equality constraints defined on a compact set. The proposed algorithm model is solved based on a hybrid-method coupling, a BBM and a gradient-based RQP method. The first phase of the optimization involves a static-BBM that achieves global optimality in solving the convex MILP model [39].

$\mathrm{BBM}$ is an efficient enumeration procedure for examining all possible integer feasible solutions. The pure ILP is relaxed to linear programming (LP), namely LP relaxations by removing the integrality conditions [39]. The concepts of branching, bounding, and fathoming are used to obtain the final solution in the process of building the enumeration tree [40]. If the LP optimum solution satisfies the integer requirement, the IP problem is solved. Otherwise, the LP objective value becomes the initial upper bound on the IP optimal value and the root node is partitioned into two successor nodes (subproblems) by two branches [39,40]. When a feasible binary integer solution is obtained via LP relaxations, an optimum point is displayed $[39,40]$. The main disadvantage is that each BBM has an alternative strategy to built the inherent-tree structure leading to a nonunique global minimum for the OPP $[39,40]$. The static strategies are best-first, depth-first, best-estimate, best-projection and breadth-first search strategy $[39,40]$. The number of LP relaxations being derived affects the PMU locations and the convergence speed justifying the differences among the derived running time by different MILP routines as shown in Table A5.

The second phase involves an RQP method, embedded in the fmincon optimizer, that generates a convergent sequence of estimated points to the global minimum point [38]. The RQP algorithm builds a sequence of non-strict global minima over the feasible region that constitutes by the objective function, the bound constraints and the constraint function.

Each optimum point satisfies the binary restriction as shown in Table A4. The RQP detects the solution point, whereas the first-order optimality measure is less than the selected tolerance without constraint violation. The objective is non-decreasing in feasible directions within the value of the function tolerance without constraint violation. Feasible directions are vectors from the current point that locally satisfy the constraints [35]. At a given point $\vec{x}$, not necessarily feasible, the algorithm generates a new point such that the first-order optimality is satisfied at the point and the step-length parameter is determined to minimize the penalty function [35]. The need for the penalty function stems from the fact that, that RQP algorithms do not maintain feasibility at each step performed by the underdetermined nonlinear programming model [35]. 
Starting from an arbitrary initial estimate, the step-length is required to enforce global convergence of the RQP method, i.e., the approximation of a point satisfying the necessary Karush-Kuhn-Tucker (KKT) optimality conditions [35]. The global convergence is included via a line-search requiring at each step the decrease of the penalty function whose reduction leads to optimality [35]. The fmincon optimizer computed the step-length to satisfy a certain Armijo condition to decrease the penalty function [38].

When the iterations are outsized, even if the iterative point is sufficiently close to the optimum point, the algorithm cannot guarantee that the step-length is 1 [35]. As shown from the simulation results, the unit step- length is achieved so that the RQP does not suffer from slow convergence near to the solution point, ensuring a superlinear convergence rate.

Hence, the Maratos Effect, where the method makes slow progress because second-order changes in the constraints are magnified and outweigh reductions in the objective function, is avoided. It is observed the fast ultimate convergence due to the full accepted step-length by the algorithm for estimates remote from the optimum point. The RQP presents both global and local convergence properties concerned with the asymptotic rate of the convergence, which indicates the rapidity with which the discrepancy between the iterates and the solution goes to zero. The number of function evaluations measures the convergence rate [35].

Provided that the function evaluations are the most time-consuming part of the algorithm, this process characterizes the convergence speed [35]. Using approximations differences, the RQP detects the solution point but takes longer to achieve optimality. This suggests that the best performance would be obtained by combining analytical given gradient constraints with RQP. The RQP requires only a small number of iterations and function evaluations to terminate successfully.

The function evaluations are significantly reduced due to the fact that the gradients are given analytically to the optimizer avoiding finite-differencing of approximate derivatives [35]. As a result, the algorithm's efficiency, measured by function, gradients evaluations and iterations, is improved concerning past studies $[11,12,16]$. Thus, the size-reducing transformation, which leads to an underdetermined nonlinear programming model, reduces the total run-time significantly as the power network size grows up regarding past studies [11,12,16].

\section{Conclusions}

This paper presents an underdetermined nonlinear programming model having fewer equality constraints than the design variables on a closed and bounded set for the solution of the optimal PMU placement problem. The proposed formulation leads to a simpler algorithm model ensuring complete power system observability compared to existing well-determined methods. The minimization model is tested on standard IEEE test systems. An RQP method is proposed to solve the proposed nonlinear model. The RQP produces a global as well as local convergence towards globally optimal solutions quickly with guaranteed accuracy. Each optimum point is a set of binary values assigned to the design variable. The strategic implications of our proposed method cover a wide range of methodological and applied contributions:

(1) It delivers a fully functional solution for the OPP problem, with efficiency and increased effectiveness.

(2) It serves as a first test best for a novel methodological approach for cost-efficient solutions to improve the monitoring of the power network across large geographic areas.

(3) It has the potential to be integrated with sensor networks and 5G networks as well as advanced Data Miners in order to promote increased performance in energy management.

(4) This approach can also be integrated with energy hardware solutions for advanced, low and middle scale smart home and smart city projects.

The items (3) and (4) above also define the future research directions for our research study. We intend to use this methodology for smart home power management applications as well as to analyze 
the impact of our approach for sophisticated data mining services for smart allocation and storage of energy resources over power grids. We understand that the computational sophistication of our approach and it's technical character can also be very useful for other researchers that are dealing with the same research problems. Last but not least the issue of Smart Grids management will require more efforts in the near future with the evolution of smart homes and smart cities applications.

Author Contributions: The paper was a collaborative effort among the authors. N.P.T., M.L. and R.B. equally contributed collectively to the theoretical analysis, modeling, simulation and manuscript preparation. All authors have read and agreed to the published version of the manuscript..

Funding: This research was funded by Special Account for Research Funding (E.L.K.E) of National Technical University of Athens (NTUA), project no.11444.

Conflicts of Interest: The authors declare no conflict of interest.

\section{Abbreviations}

$\begin{array}{ll}\text { SCADA } & \text { Supervisory Control and Data Acquisition } \\ \text { WAMS } & \text { Wide Area Monitoring System } \\ \text { SE } & \text { State Estimator } \\ \text { RTU } & \text { Remote Terminal Unit } \\ \text { OPP } & \text { Optimal PMU Placement } \\ \text { PMU } & \text { Phasor Measurement Unit } \\ \text { ILP } & \text { Integer Linear Programming } \\ \text { BILP } & \text { Binary Integer Linear Programming } \\ \text { MILP } & \text { Mixed Integer Linear Programming } \\ \text { LP } & \text { Linear Programming } \\ \text { NLP } & \text { Nonlinear Programming } \\ \text { KKT } & \text { Karush-Kuhn-Tucker } \\ \text { BFGS } & \text { Broydon-Fletcher-Goldfarbo-Shanno } \\ \text { BBM } & \text { Branch-and-Bound Method } \\ \text { RQP } & \text { Recursive Quadratic Programming } \\ \text { intlinprog } & \text { Integer Linear Programming solver } \\ \text { SCIP } & \text { Solve Constraint Integer Program solver }\end{array}$

\section{Appendix A}

Table A1. Optimal Phasor Measurement Unit (PMU) locations for IEEE 14-, 30-, 57-, 118- bus systems.

\begin{tabular}{|c|c|c|c|c|}
\hline \multirow{3}{*}{ IEEE System } & \multicolumn{4}{|c|}{ ILP Routines for Solving the Pure Constraint Integer Linear Problem } \\
\hline & Gurobi & MOSEK & Intlinprog & SCIP \\
\hline & \multicolumn{4}{|c|}{ Optimal PMU Locations } \\
\hline 14 bus & $2,7,10,13$ & $2,6,7,9$ & $2,8,10,13$ & $2,7,11,13$ \\
\hline 30 bus & $1,5,6,9,10,12,15,19,25,27$ & $1,5,8,9,10,12,18,23,25,30$ & $1,5,8,10,11,12,19,23,26,29$ & $2,4,6,9,10,12,15,19,25,30$ \\
\hline 57 bus & $\begin{array}{l}1,6,9,15,19,20,24,25,28 \\
32,36,38,39\end{array}$ & $\begin{array}{l}1,2,6,13,19,22,25,27,32 \\
36,41,43,47,51,52,55\end{array}$ & $\begin{array}{l}1,4,9,20,23,27,29,30,32 \\
36,38,41,45,46,50,54\end{array}$ & $2,6,12,14,19,22,25,27,32$ \\
\hline 118 bus & $\begin{array}{l}1,5,9,12,15,17,21,23,28 \\
30,36,40,44,46,50,51,54 \\
62,63,68,71,75,77,80,85 \\
86,91,94,102,105,110,114\end{array}$ & $\begin{array}{c}1,6,9,11,12,17,21,25,29 \\
34,37,41,45,49,53,56,62 \\
63,68,71,72,75,77,80,85 \\
86,90,94,102,105,110,114\end{array}$ & $\begin{array}{c}2,5,10,12,15,17,21,25,29 \\
34,37,41,45,49,53,56,62 \\
64,72,73,75,77,80,85,87 \\
91,94,101,105,110,114,116\end{array}$ & $\begin{array}{l}3,5,9,11,12,17,21,25,28 \\
34,37,40,45,49,52,56,62 \\
63,68,70,71,75,77,80,85 \\
86,90,94,101,105,110,114\end{array}$ \\
\hline
\end{tabular}


Table A2. Optimal PMU locations for IEEE 300- bus systems.

Optimal PMU Locations of PMUs using SCIP

$1,2,3,11,12,15,17,22,23,25,26,27,33,37,38,43,48,49,53,54,55,58,59,60,62,64,65,68,71,73,79,83,85,86$, $88,92,93,98,99,101,109,111,112,113,116,118,119,124,132,133,138,139,143,145,152,157,160,163,173,177$, $183,187,189,190,193,196,200,204,208,210,211,213,216,217,219,222,225,228,267,268,269,270,272,273,274$, 276,294

\section{Optimal Locations of PMUs Through Intlinprog of MATLAB Optimization Toolbox}

$1,2,3,11,15,21,23,25,27,30,33,37,38,41,43,48,49,53,54,64,68,69,71,79,83,86,88,93,96,98,99,101,109$, $111,112,113,116,119,128,132,135,139,141,152,157,160,164,170,183,187,188,189,190,193,196,202,209,210$, $212,215,216,217,222,224,228,230,233,236,237,238,240,242,251,252,253,262,264,265,268,269,270,272,275$,

$276,277,299,300$

Optimal Locations of PMUs using Gurobi

$1,2,3,11,12,15,17,20,22,23,25,27,29,33,37,38,43,48,49,53,54,55,58,59,60,62,64,65,68,71,79,83,85,86$, $88,89,93,98,99,101,109,111,112,113,116,118,119,124,132,133,138,139,143,145,152,157,163,167,168,173$, $183,184,189,190,193,196,200,204,208,210,211,213,216,217,219,224,225,228,267,268,269,270,272,273,274$,

276,294

\section{Optimal Locations of PMUs using MOSEK}

$1,2,3,11,12,13,15,17,22,23,25,27,30,33,37,38,43,48,49,53,54,55,58,59,60,62,64,65,68,71,76,80,85,86$, $88,92,93,96,98,99,101,109,111,112,113,116,118,122,125,132,135,139,141,145,152,157,160,163,171,173$, $183,187,188,189,190,193,196,202,208,210,211,213,216,217,219,222,226,229,267,268,269,270,272,273,274$, 276, 294

Table A3. Simulation results on underdetermined system of equations.

\begin{tabular}{|c|c|c|c|c|c|c|}
\hline \multicolumn{2}{|c|}{ IteF-Count } & $f(x)$ & \multirow{2}{*}{$\begin{array}{c}\text { Feasibility } \\
9.861 \times 10^{-1}\end{array}$} & \multirow[t]{2}{*}{ Steplength } & \multirow[t]{2}{*}{ Norm of Step } & \multirow{2}{*}{$\begin{array}{c}\text { First-Order } \\
\text { Optimality } \\
2.000\end{array}$} \\
\hline 0 & 1 & $1.032236 \times 10^{2}$ & & & & \\
\hline 1 & 2 & $9.666889 \times 10^{1}$ & $9.984 \times 10^{-1}$ & 1.000 & 4.379 & $4.059 \times 10^{2}$ \\
\hline 2 & 3 & $9.931676 \times 10^{1}$ & $2.984 \times 10^{-1}$ & 1.000 & 2.952 & $4.062 \times 10^{2}$ \\
\hline 3 & 4 & $9.914983 \times 10^{1}$ & $1.361 \times 10^{-1}$ & $7.000 \times 10^{-1}$ & 1.644 & $4.062 \times 10^{2}$ \\
\hline 4 & 5 & $1.032552 \times 10^{2}$ & $4.321 \times 10^{-2}$ & 1.000 & $9.177 \times 10^{-1}$ & $4.060 \times 10^{2}$ \\
\hline 5 & 6 & $1.024552 \times 10^{2}$ & $1.324 \times 10^{-2}$ & 1.000 & 1.406 & $4.056 \times 10^{2}$ \\
\hline 6 & 7 & $1.023897 \times 10^{2}$ & $6.082 \times 10^{-3}$ & $7.000 \times 10^{-1}$ & $8.273 \times 10^{-1}$ & $4.060 \times 10^{2}$ \\
\hline 7 & 8 & $1.033093 \times 10^{2}$ & $6.327 \times 10^{-4}$ & 1.000 & $9.285 \times 10^{-1}$ & $4.062 \times 10^{2}$ \\
\hline 8 & 9 & $1.022358 \times 10^{2}$ & $3.298 \times 10^{-6}$ & 1.000 & $4.972 \times 10^{-1}$ & $4.062 \times 10^{2}$ \\
\hline 9 & 10 & $9.735171 \times 10^{1}$ & $1.030 \times 10^{-7}$ & 1.000 & 1.702 & $4.062 \times 10^{2}$ \\
\hline 10 & 11 & $9.510909 \times 10^{1}$ & $2.575 \times 10^{-8}$ & 1.000 & 1.369 & $4.062 \times 10^{2}$ \\
\hline 11 & 12 & $9.345127 \times 10^{1}$ & $6.438 \times 10^{-9}$ & 1.000 & $8.949 \times 10^{-1}$ & $4.062 \times 10^{2}$ \\
\hline 12 & 13 & $9.241002 \times 10^{1}$ & $1.610 \times 10^{-9}$ & 1.000 & $7.573 \times 10^{-1}$ & $4.060 \times 10^{2}$ \\
\hline 13 & 14 & $9.202713 \times 10^{1}$ & $4.024 \times 10^{-10}$ & 1.000 & $6.289 \times 10^{-1}$ & $4.062 \times 10^{2}$ \\
\hline 14 & 15 & $9.200234 \times 10^{1}$ & $7.112 \times 10^{-19}$ & 1.000 & $1.842 \times 10^{-1}$ & $4.061 \times 10^{2}$ \\
\hline 15 & 16 & $9.193331 \times 10^{1}$ & 0.000 & 1.000 & $2.631 \times 10^{-2}$ & $4.061 \times 10^{2}$ \\
\hline 16 & 17 & $9.157585 \times 10^{1}$ & 0.000 & 1.000 & $1.001 \times 10^{-1}$ & $4.061 \times 10^{2}$ \\
\hline 17 & 18 & $8.991064 \times 10^{1}$ & 0.000 & 1.000 & $5.182 \times 10^{-1}$ & $4.059 \times 10^{2}$ \\
\hline 18 & 19 & $8.738703 \times 10^{1}$ & 0.000 & 1.000 & 1.735 & $4.897 \times 10^{2}$ \\
\hline 19 & 20 & $8.705728 \times 10^{1}$ & $1.110 \times 10^{-16}$ & 1.000 & $6.643 \times 10^{-1}$ & $4.931 \times 10^{2}$ \\
\hline 20 & 21 & $8.701730 \times 10^{1}$ & $2.220 \times 10^{-16}$ & 1.000 & $2.365 \times 10^{-1}$ & $4.918 \times 10^{2}$ \\
\hline 21 & 22 & $8.700852 \times 10^{1}$ & $2.220 \times 10^{-16}$ & 1.000 & $9.617 \times 10^{-2}$ & $4.923 \times 10^{2}$ \\
\hline 22 & 23 & $8.700622 \times 101$ & $2.190 \times 10^{-16}$ & 1.000 & $7.026 \times 10^{-2}$ & $4.921 \times 10^{2}$ \\
\hline 23 & 24 & $8.700109 \times 10^{1}$ & $2.220 \times 10^{-16}$ & 1.000 & $6.787 \times 10^{-2}$ & $4.915 \times 10^{2}$ \\
\hline 24 & 25 & $8.700013 \times 10^{1}$ & $2.220 \times 10^{-16}$ & 1.000 & $3.499 \times 10^{-2}$ & $4.915 \times 10^{2}$ \\
\hline 25 & 26 & $8.700002 \times 10^{1}$ & $2.220 \times 10^{-16}$ & 1.000 & $1.208 \times 10^{-2}$ & $4.915 \times 10^{2}$ \\
\hline 26 & 27 & $8.700000 \times 10^{1}$ & $1.110 \times 10^{-16}$ & 1.000 & $4.134 \times 10^{-3}$ & $4.915 \times 10^{2}$ \\
\hline 27 & 28 & $8.700000 \times 10^{1}$ & $1.110 \times 10^{-16}$ & 1.000 & $1.391 \times 10^{-3}$ & $4.914 \times 10^{2}$ \\
\hline 28 & 29 & $8.700000 \times 10^{1}$ & $2.220 \times 10^{-16}$ & 1.000 & $5.724 \times 10^{-4}$ & $4.914 \times 10^{2}$ \\
\hline
\end{tabular}


Table A3. Cont.

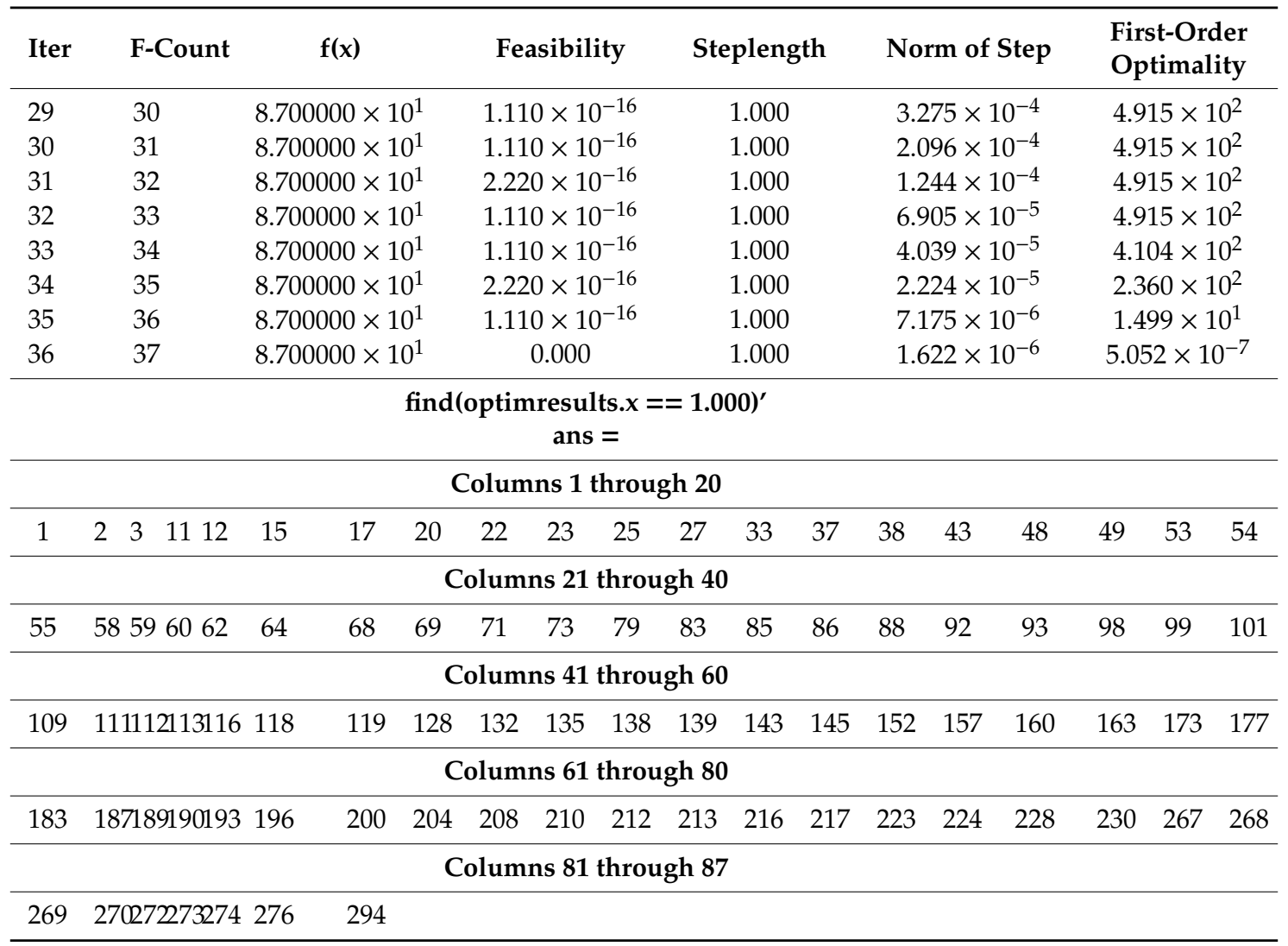

Table A4. Simulation results using the Recursive Quadratic Programming (RQP) method.

\begin{tabular}{|c|c|c|}
\hline Test System & $\begin{array}{l}\text { Objective } \\
\text { Value }\end{array}$ & Optimal PMU Placement \\
\hline IEEE-14 bus & 4 & $\begin{array}{c}2,8,10,13 \\
2,6,8,9 \\
2,7,11,13 \\
2,7,10,13 \\
2,6,7,9\end{array}$ \\
\hline IEEE-30 bus & 10 & $\begin{array}{c}1,7,8,10,11,12,18,24,26,27 \\
1,7,8,10,11,12,18,24,25,27 \\
1,7,8,10,11,12,18,24,26,27 \\
1,7,8,9,10,12,18,24,26,27 \\
1,6,7,9,10,12,18,23,26,27 \\
1,7,8,9,10,12,15,20,25,27 \\
1,7,8,10,11,12,18,24,25,27\end{array}$ \\
\hline IEEE-57 bus & 17 & $\begin{array}{c}2,6,12,19,22,25,27,29,32,36,38,41,45,46,50,54,57 \\
2,6,12,14,19,22,25,27,32,36,41,45,47,50,52,55,57 \\
1,4,6,10,19,22,25,27,32,36,39,41,44,46,49,52,55 \\
1,4,6,13,20,23,25,27,32,36,41,44,47,51,52,55,57 \\
1,4,6,10,19,22,25,27,32,36,41,44,46,49,52,55,57 \\
1,4,9,10,19,22,25,26,29,32,36,39,41,44,46,49,53 \\
1,4,6,10,20,23,27,30,32,36,41,44,46,49,52,55,57 \\
1,6,9,15,19,22,25,27,32,36,38,39,41,47,50,52,55 \\
2,6,12,14,19,22,25,27,32,36,41,44,47,50,52,54,57 \\
86,91,94,102,105,110,114,116 \\
3,5,10,12,13,17,21,25,28,34,37,41,49,53,76,77,80,85, \\
3,5,10,12,15,17,21,24,26,28,34,37,40,45,49,53,56,62,64,73,75,77,80,85, \\
86,90,94,102,105,110,114,116 \\
3,5,9,12,13,17,21,25,29,34,37,41,45,49,53,56,62,64,72,73,75,77,80,85,87, \\
90,94,102,105,110,114,116 \\
1,5,9,11,12,17,21,24,26,28,34,37,41,45,49,53,56,62,64,73,75,77,80,85,87, \\
90,94,102,105,110,114,116 \\
2,5,10,11,12,17,21,23,29,30,34,37,40,45,49,53,56,62,63,68,71,75,77,80, \\
85,87,90,94,102,105,110,115\end{array}$ \\
\hline
\end{tabular}


Table A4. Cont.

\begin{tabular}{|c|c|c|}
\hline Test System & $\begin{array}{l}\text { Objective } \\
\text { Value }\end{array}$ & Optimal PMU Placement \\
\hline & & $\begin{array}{r}2,5,9,12,15,17,21,24,26,28,34,37,41,45,49,53,56,62,64,68,73,75,77,80,85, \\
87,91,94,102,105,110,114 \\
1,5,9,11,12,17,21,24,26,28,34,37,41,45,49,53,56,62,64,68,73,75,77,80,85, \\
87,91,94,102,105,110,114 \\
3,5,10,12,13,17,21,25,28,34,37,41,45,49,53,56,62,63,68,70,71,76,79,84, \\
87,89,92,96,100,105,110,114 \\
3,5,10,12,13,17,21,25,29,34,37,40,45,49,53,56,62,63,68,70,71,78,85,86, \\
91,92,96,100,105,110,114,118 \\
3,5,10,12,13,17,21, \begin{array}{r}25,28,34,37,40,45,49,53,56,62,63,68,70,71,78,84,86, \\
89,92,96,100,105,110,114,118\end{array} \\
1,5,10,12,13,17,21,25,29,34,37,40,45,49,53,56,62,63,68,70,71,78,84,86, \\
89,92,96,100,105,110,114,118\end{array}$ \\
\hline IEEE-300 bus & 87 & 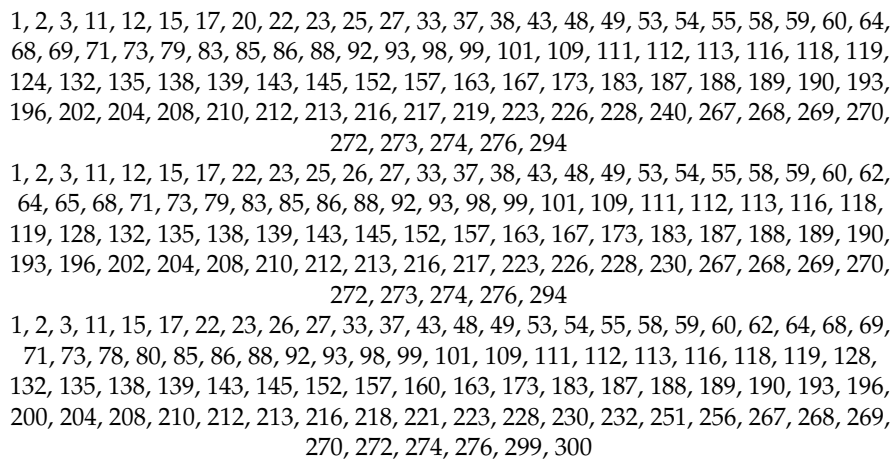 \\
\hline IEEE-300 bus & 87 & 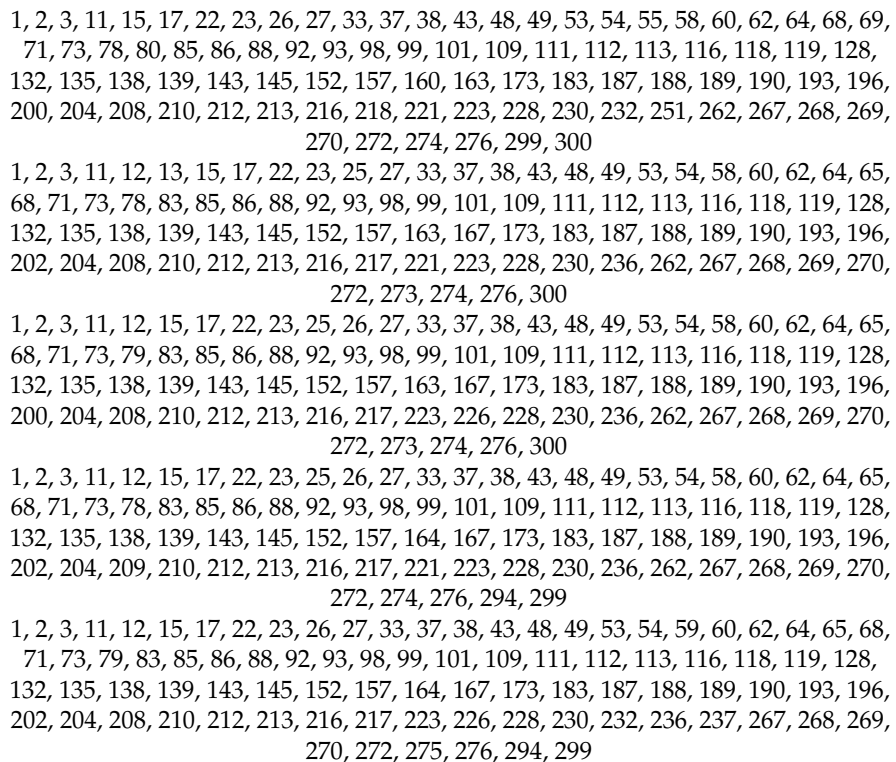 \\
\hline
\end{tabular}

Table A5. Required PMU numbers and elapsed computational time.

\begin{tabular}{cccccccc}
\hline \multirow{2}{*}{$\begin{array}{c}\text { IEEE } \\
\text { System }\end{array}$} & \multirow{2}{*}{$\begin{array}{c}\text { Optimal } \\
\text { Value }\end{array}$} & \multicolumn{3}{c}{ MIXED-INTEGER LINEAR PROGRAM } & \multicolumn{2}{c}{ NONLINEAR PROGRAM } \\
\cline { 3 - 7 } & & \multicolumn{4}{c}{ Elapsed Time (s) } & \multicolumn{2}{c}{ Average Elapsed Time (s) } \\
\cline { 3 - 7 } & & Gurobi & MOSEK & Intlinprog & SCIP & $\begin{array}{c}\text { Analytically } \\
\text { Gradients }\end{array}$ & $\begin{array}{c}\text { Approximations } \\
\text { Differences }\end{array}$ \\
\hline 14 bus & 4 & 0.02417 & 0.11 & 0.050035 & 0.01873 & 0.087140 & 0.322370 \\
30 bus & 10 & 0.01506 & 0.08 & 0.018850 & 0.04615 & 0.151758 & 0.343953 \\
57 bus & 17 & 0.02302 & 0.17 & 0.038206 & 0.02890 & 0.493773 & 1.608577 \\
118 bus & 32 & 0.02459 & 0.16 & 0.036103 & 0.04214 & 0.249894 & 3.347825 \\
300 bus & 87 & 0.04447 & 0.17 & 0.458907 & 0.05359 & 2.703687 & 56.31137 \\
\hline
\end{tabular}




\section{References}

1. Phadke, A.G.; Thorp, J.S. Synchronized Phasor Measurements and Their Applications, 2nd ed.; Springer: New York, NY, USA, 2017.

2. Manousakis, N.M.; Korres, G.N.; Georgilakis, P.S. Taxonomy of PMU placement methodologies. IEEE Trans. Power Syst. 2012, 27, 1070-1077. [CrossRef]

3. Nazari-Heris, M.; Mohammadi-Ivatloo, B. Application of heuristic algorithms to optimal PMU placement in electric power systems: An updated review. Renew. Sustain. Energy Rev. 2015, 50, 214-228. [CrossRef]

4. Babu, R.; Bhattacharyya, B. An Approach for Optimal Placement of Phasor Measurement Unit for Power Network Observability Considering Various Contingencies. Iran. J. Sci. Technol. Trans. Electr. Eng. 2018, 42, 161-183. [CrossRef]

5. Rahman, N.H.A.; Zobaa, A.F. Optimal PMU placement using topology transformation method in power systems. J. Adv. Res. 2016, 7, 625-634. [CrossRef]

6. Poirion, P.L.; Toubaline, S.; D'Ambrosio, C.; Liberti, L. The power edge set problem. Networks 2016, 68, 104-120. [CrossRef]

7. Pal, A.; Vullikanti, A.K.S.; Ravi, S.S. A PMU Placement Scheme Considering Realistic Costs and Modern Trends in Relaying. IEEE Trans. Power Syst. 2017, 32, 552-561. [CrossRef]

8. Pal, A.; Sánchez, A.G.A.; Thorp, J.S.; Centeno, V.A. A Community-based Partitioning Approach for phasor Measurement Unit Placement in Large systems. Electr. Power Compon. Syst. 2016, 44, 1317-1329. [CrossRef]

9. Theodorakatos, N.P. Optimal Phasor Measurement Unit Placement for Numerical Observability Using a Two-Phase Branch-and-Bound Algorithm. Int. J. Emerg. Electr. Power Syst. 2018, 19, 1-25. [CrossRef]

10. Shahriar, M.S.; Habiballah, I.O.; Hussein, H. Optimization of Phasor Measurement Unit (PMU) Placement in Supervisory Control and Data Acquisition (SCADA)-Based Power System for Better State-Estimation Performance. Energies 2018, 11, 570. [CrossRef]

11. Manousakis, N.M.; Korres, G.N. A Weighted Least Squares Algorithm for optimal PMU placement. IEEE Trans. Power Syst. 2013, 28, 3499-3500. [CrossRef]

12. Theodorakatos, N.P.; Manousakis, N.M.; Korres, G.N. Optimal Placement of Phasor Measurement Units with Linear and Non-linear Models. Electr. Power Compon. Syst. 2015, 43, 357-373. [CrossRef]

13. Korres, G.N.; Manousakis, N.M.; Xygkis, T.C.; Lofberg, J. Optimal phasor measurement unit placement for numerical observability in the presence of conventional measurement using semi-definite programming. IET Gener. Transm. Distrib. 2015, 9, 2427-2436. [CrossRef]

14. Manousakis, N.M.; Korres, G.N. Optimal Allocation of Phasor Measurement Units Considering Various Contingencies and Measurement Redundancy. IEEE Transcations Instrum. Meas. 2019. [CrossRef]

15. Almunif, A.; Fan, L. DC State Estimation Model-Based Mixed Integer Semidefinite Programming for Optimal PMU Placement. In Proceedings of the 2018 North American Power Symposium (NAPS), Fargo, ND, USA, 9-11 September 2018; pp. 1-6.

16. Almunif, A.; Fan, L. Mixed integer linear programming and nonlinear programming for optimal PMU placement. In Proceedings of the 2017 North American Power Symposium (NAPS), Morgantown, WV, USA, 17-19 September 2017; pp. 1-6.

17. Almunif, A.; Fan, L. Optimal PMU placement for modeling power grid observability with mathematical programming methods. Int. Trans. Electr. Energ. Syst. 2020, 30, e12182. [CrossRef]

18. Koutsoukis, N.C.; Manousakis, N.M.; Georgilakis, P.S.; Korres, G.N. Numerical observability method for optimal phasor measurement units placement using recursive Tabu search method. IET Gener. Transm. Distrib. 2013, 7, 347-356. [CrossRef]

19. Babu, R.; Bhattacharyya, B. Optimal allocation of phasor measurement unit for full observability of the connected power network. Electr. Power Energy Syst. 2016, 79, 89-97. [CrossRef]

20. Mishra, C.; Jones, K.D.; Pal, A.; Centeno, V. Binary particle swarm optimisation-based optimal substation coverage algorithm for phasor measurement unit installations in practical systems. IET Gener. Transm. Distrib. 2016, 10, 1-8. [CrossRef]

21. Singh, S.P. A Multi-objective PMU Placement Method in Power System via Binary Gravitational Search Algorithm. Electr. Power Compon. Syst. 2017, 45, 1-14. [CrossRef] 
22. Dalali, M.; Karegar, H.K. Optimal PMU placement for full observability of the power network with maximum redundancy using modified binary cuckoo optimisation algorithm. IET Gener. Transm. Distrib. 2016, 10, 2817-2824. [CrossRef]

23. Müller, H.H.; Castro, C.A. Genetic algorithm-based phasor measurement unit placement method considering observability and security criteria. IET Gener. Transmiss. Distrib. 2016, 10, 270-280. [CrossRef]

24. Theodorakatos, N.P. Optimal Phasor Measurement Unit Placement for Numerical Observability Using Branch-and-Bound and a Binary-Coded Genetic Algorithm. Electr. Power Compon. Syst. 2019, 47, 357-371.

25. Wilson, R.E.; Zevenbergen, G.A.; Mah, D.L.; JayMurphy, A. Calculation of transmission line parameters from synchronized measurements. Electr. Mach. Power Syst. 1999, 27, 1269-1278.

26. Abdulrahman, K.; Al-Othman El-Naggar, K.M.; AlSharidah, M.E. On-line Estimation of Transmission Line Parameters Using Synchronized Measurements. Electr. Power Compon. Syst. 2016, 44, 233-239.

27. Manousakis, N.M.; Korres, G.N. A hybrid power system state estimator using synchronized and unsynchronized sensors. Int. Trans. Electr. Energ. Syst. 2018, 28, e2580. [CrossRef]

28. Alexopoulos, T.A.; Manousakis, N.M.; Korres, G.N. Fault Location Observability using Phasor Measurements Units via Semidefinite Programming. IEEE Access 2016, 4, 5187-5195. [CrossRef]

29. Theodorakatos, N.P. Fault Location Observability Using Phasor Measurement Units in a Power Network Through Deterministic and Stochastic Algorithms. Electr. Power Compon. Syst. 2019, 47, 212-229. [CrossRef]

30. Kavasseri, R.; Srinivasan, S.K. Joint placement of phasor and conventional power flow measurements for fault observability of power systems. IET Gener. Transm. Distrib. 2011, 5, 1019-1024. [CrossRef]

31. Zhang, Y.; Li, T.; Na, G.; Li, G.; Li, Y. Optimized Extreme Learning Machine for Power System Transient Stability Prediction Using Synchrophasors. Math. Probl. Eng. 2015, 2015, 529724. [CrossRef]

32. Baldik, R. Applied Optimization: Formulation and Algorithms for Engineering Systems; Cambridge University Press: Cambridge, UK, 2006.

33. Williams, H.P. Model Building in Mathematical Programming; John Wiley \& Sons: New York, NY, USA, 2013.

34. Puranik, Y.; Sahinidis, N.V. Deletion Presolve for Accelerating Infeasibility Diagnosis in Optimization Models. Inf. J. Comput. 2017, 29, 754-766. [CrossRef]

35. Arora, J.S. Introduction to Optimum Design, 4th ed.; Elsevier Inc.: Amsterdam, The Netherlands, 2016.

36. Chinneck, J.W. Feasibility and Infeasibility in Optimization: Algorithms and Computational Methods; Springer, International Series in Operations Research \& Management Science: Cham, Switzerland, 2008.

37. Maratos, N.G. Exact Penalty Function Algorithms for Finite Dimensional and Optimization Problems. Ph.D. Thesis, Imperial College of Science and Technology, University of London, London, UK, 1978.

38. The MathWorks Inc. Optimization Toolbox for use with MATLAB R ${ }^{\circledR}$. User's Guide for Mathwork. 2016. Available online: www.mathworks.com (accessed on 1 February 2019).

39. Karlof, J.K. Integer Programming: Theory and Practice; CRC Press, Taylor \& Francis: Boca Raton, FL, USA, 2006.

40. Cormen, T.H.; Leiserson, C.E.; Rivest, R.L.; Stein, C. Introduction to Algorithms; Massachusetts Institute of Technology; The MIT Press: Cambridge, MA, USA; London, UK, 2009.

41. Berthold, T.; Gamrath, G.; Gleixner, A.M.; Heinz, S.; Koch, T.; Shinano, Y. Solving mixed integer linear and nonlinear problems using the SCIP optimization suite. ZIB-Rep. 2012, 12-27, 1-23.

42. Parametric Fusion (MOSEK 9.2). Available online: https://www.mosek.com (accessed on 4 March 2019).

43. The Fastest Solver. Available online: http://www.gurobi.com (accessed on 10 August 2019).

44. Electrical \& Computer Engineering. Available online: http://www.ee.washington.edu/research/pstca (accessed on 12 July 2019).

(C) 2020 by the authors. Licensee MDPI, Basel, Switzerland. This article is an open access article distributed under the terms and conditions of the Creative Commons Attribution (CC BY) license (http://creativecommons.org/licenses/by/4.0/). 